\title{
DARK ENERGY - DARK MATTER UNIFICATION: GENERALIZED CHAPLYGIN GAS MODEL*
}

\author{
ORFEU BERTOLAMI \\ Instituto Superior Técnico, Departamento de Física \\ Av. Rovisco Pais, 1049-001 \\ Lisboa, Portugal \\ E-mail: orfeu@cosmos.ist.utl.pt
}

\begin{abstract}
We review the main features of the generalized Chaplygin gas (GCG) proposal for unification of dark energy and dark matter and discuss how it admits an unique decomposition into dark energy and dark matter components once phantom-like dark energy is excluded. In the context of this approach we consider structure formation and show that unphysical oscillations or blow-up in the matter power spectrum are not present. Moreover, we demonstrate that the dominance of dark energy occurs about the time when energy density fluctuations start evolving away from the linear regime.
\end{abstract}

\section{Introduction}

The GCG model ${ }^{1,2}$ is an interesting alternative to more conventional approaches for explaining the observed accelerated expansion of the Universe such as a cosmological constant ${ }^{3}$ or quintessence ${ }^{4}$. It is worth remarking that quintessence is related to the idea that the cosmological term could evolve $^{5}$ and with attempts to tackle the cosmological constant problem.

In the GCG approach one considers an exotic equation of state to describe the background fluid:

$$
p_{c h}=-\frac{A}{\rho_{c h}^{\alpha}},
$$

where $A$ and $\alpha$ are positive constants. The case $\alpha=1$ corresponds to the Chaplygin gas. In most phenomenological studies the range $0<\alpha \leq 1$

*Talk presented at the V New Worlds in Astroparticle Physics Conference, Faro, Portugal, 8-10 January 2005. 
is considered. Within the framework of Friedmann-Robertson-Walker cosmology, this equation of state leads, after being inserted into the relativistic energy conservation equation, to an evolution of the energy density $a^{2}$

$$
\rho_{c h}=\left[A+\frac{B}{a^{3(1+\alpha)}}\right]^{\frac{1}{1+\alpha}},
$$

where $a$ is the scale-factor of the Universe and $B$ a positive integration constant. From this result, one can understand a striking property of the GCG: at early times the energy density behaves as matter while at late times it behaves like a cosmological constant. This dual role is what essentially allows for the interpretation of the GCG model as an entangled mixture of dark matter and dark energy.

The GCG model has been successfully confronted with different classes of phenomenological tests: high precision Cosmic Microwave Background Radiation data ${ }^{6}$, supernova data ${ }^{7}$, and gravitational lensing ${ }^{8}$. More recently, it has been shown using the latest supernova data ${ }^{9}$, that the GCG model is degenerate with a dark energy model with a phantom-like equation of state ${ }^{10,11}$. Furthermore, it can be shown that this does not require invoking the unphysical condition of violating the dominant energy condition and does not lead to the big rip singularity in future ${ }^{10}$. It is a feature of GCG model, that it can mimic a phantom-like equation of state, but without any kind of pathologies as asymptotically the GCG approaches to a well-behaved de-Sitter universe. Structure formation has been studied in Refs. [2, 12]. In Ref. [13], the results of the various phenomenological tests on the GCG model are summarized.

Despite these pleasing performance concerns about such an unified model were raised in the context of structure formation. Indeed, it has been pointed out that one should expect unphysical oscillations or even an exponential blow-up in the matter power spectrum at present ${ }^{14}$. This difficult arises from the behaviour of the sound velocity through the GCG. Although, at early times, the GCG behaves like dark matter and its sound velocity is vanishingly small as one approaches the present, the GCG starts behaving like dark energy with a substantial negative pressure yielding a large sound velocity which, in turn, produces oscillations or blow-up in the power spectrum. In any unified approach this is inevitable unless the dark matter and the dark energy components of the fluid can be properly identified. These components are, of course, interacting as both are entangled within a single fluid. However, it can be shown that the GCG is a unique 
mixture of interacting dark matter and a cosmological constant-like dark energy, once one excludes the possibility of phantom-type dark energy ${ }^{15}$. It can be shown that due to the interaction between the components, there is a flow of energy from dark matter to dark energy. This energy transfer is vanishingly small until recent past, resulting in a negligible contribution at the time of gravitational collapse $\left(z_{c} \simeq 10\right)$. This feature makes the model indistinguishable from a CDM dominated Universe till recent past. Subsequently, just before present $(z \simeq 2)$, the interaction starts to grow yielding a large energy transfer from dark matter to dark energy, which leads to the dominance of the latter at present. Moreover, it is shown that the epoch of dark energy dominance occurs when dark matter perturbations start deviating from its linear behaviour and that the Newtonian equations for small scale perturbations for dark matter do not involve any $k$-dependent term. Thus, neither oscillations nor blow-up in the power spectrum do develop.

\section{Decomposition of the GCG fluid}

In Ref. [2], it is shown that the GCG can be described through a complex scalar field whose Lagrangian density can be written as a generalized BornInfeld theory:

$$
L_{G B I}=-A^{\frac{1}{1+\alpha}}\left[1-\left(g^{\mu \nu} \theta_{, \mu} \theta_{, \nu}\right)^{\frac{1+\alpha}{2 \alpha}}\right]^{\frac{\alpha}{1+\alpha}},
$$

which reduces into the Born-Infeld Lagrangian density for $\alpha=1$. The field $\theta$ corresponds to the phase of the complex scalar field ${ }^{2}$.

Let us now consider the decomposition of the GCG into components. Introducing the redshift dependence and using Eqs. (1) and (2), the pressure is given by

$$
p_{c h}=-\frac{A}{\left[A+B(1+z)^{3(1+\alpha)}\right]^{\frac{\alpha}{1+\alpha}}}
$$

while the total energy density can be written as

$$
\rho_{c h}=\left[A+B(1+z)^{3(1+\alpha)}\right]^{\frac{1}{1+\alpha}},
$$

where the present value of the scale-factor, $a_{0}$, has been set to 1 .

We decompose the energy density into a pressure-less dark matter component, $\rho_{d m}$, and a dark energy component, $\rho_{X}$, with an equation of state 
$p_{X}=w_{X} \rho_{X}$; hence the equation of state parameter of the GCG can be written as

$$
w=\frac{p_{c h}}{\rho_{c h}}=\frac{p_{X}}{\rho_{d m}+\rho_{X}}=\frac{w_{X} \rho_{X}}{\rho_{d m}+\rho_{X}} .
$$

Therefore, from Eqs. (4), (5) and (6), one obtains for $\rho_{X}$

$$
\rho_{X}=-\frac{\rho_{d m}}{1+w_{X}\left[1+\frac{B}{A}(1+z)^{3(1+\alpha)}\right]} .
$$

From this equation one can see that requiring that $\rho_{X} \geq 0$ leads to the constraint $w_{X} \leq 0$ for early times $(z \gg 1)$ and $w_{X} \leq-1$ for the future $(z=-1)$. Thus, one can conclude that $w_{X} \leq-1$ for the entire history of the Universe. The case $w_{X}<-1$ corresponds to the so-called phantom-like dark energy, which violates the dominant-energy condition and leads to an ill defined sound velocity (see however Ref. [10]). Excluding this possibility, then the energy density can be uniquely split as

$$
\rho=\rho_{d m}+\rho_{\Lambda}
$$

where

$$
\rho_{d m}=\frac{B(1+z)^{3(1+\alpha)}}{\left[A+B(1+z)^{3(1+\alpha)}\right]^{\frac{\alpha}{1+\alpha}}},
$$

and

$$
\rho_{\Lambda}=-p_{\Lambda}=\frac{A}{\left[A+B(1+z)^{3(1+\alpha)}\right]^{\frac{\alpha}{1+\alpha}}},
$$

from which one finds the scaling behaviour of the energy densities

$$
\frac{\rho_{d m}}{\rho_{\Lambda}}=\frac{B}{A}(1+z)^{3(1+\alpha)} .
$$

In what follows we express parameters A and B in terms of cosmological observables. From Eqs. (9) and (10), it implies that

$$
\rho_{c h 0}=\rho_{d m 0}+\rho_{\Lambda 0}=(A+B)^{\frac{1}{1+\alpha}},
$$




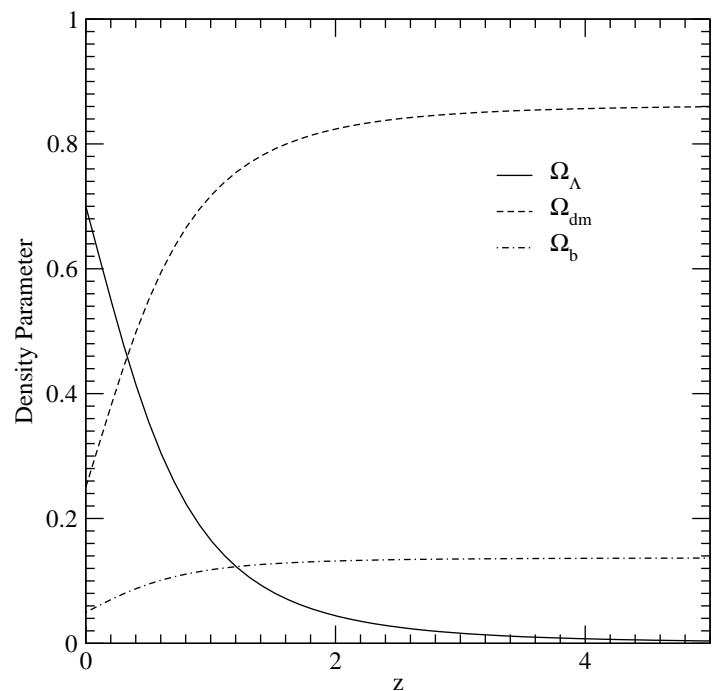

Figure 1. Density parameters $\Omega_{d m}$ and $\Omega_{\Lambda}$ and $\Omega_{b}$ as a function of redshift. It is assumed that $\Omega_{b 0}=0.05, \Omega_{d m 0}=0.25, \Omega_{\Lambda 0}=0.7$ and $\alpha=0.2$.

where $\rho_{c h 0}, \rho_{d m 0}$ and $\rho_{\Lambda 0}$ are the present values of $\rho_{c h}, \rho_{m}$ and $\rho_{\Lambda}$, respectively. Constants $A$ and $B$ can then be written as a function of $\rho_{c h 0}$

$$
A=\rho_{\Lambda 0} \rho_{c h 0}^{\alpha} ; \quad B=\rho_{d m 0} \rho_{c h 0}^{\alpha} .
$$

It is also interesting to express $A$ and $B$ in terms of $\Omega_{d m 0}, \Omega_{\Lambda 0}$, the present values of the fractional energy densities $\Omega_{d m(\Lambda)}=\rho_{m(\Lambda)} / \rho_{c}$ where $\rho_{c}$ is the critical energy density, $\rho_{c}=3 H^{2} / 8 \pi G$. From the Friedmann equation

$$
3 H^{2}=8 \pi G\left[A+B(1+z)^{3(1+\alpha)}\right]^{\frac{1}{1+\alpha}}+8 \pi G \rho_{b 0}(1+z)^{3}
$$

where $\rho_{b 0}$ is the baryon energy density at present, one obtains

$$
A \simeq \Omega_{\Lambda 0} \rho_{c 0}^{(1+\alpha)}, B \simeq \Omega_{d m 0} \rho_{c 0}^{(1+\alpha)} .
$$

Therefore, with the present value of the Hubble parameter, $H_{0}$ : 


$$
H^{2}=H_{0}^{2}\left[\left[\Omega_{\Lambda 0}+\Omega_{d m 0}(1+z)^{3(1+\alpha)}\right]^{\frac{1}{1+\alpha}}+\Omega_{b 0}(1+z)^{3}\right]
$$

one can write the fractional energy densities $\Omega_{d m}, \Omega_{\Lambda}$ and $\Omega_{b}$ as

$$
\begin{gathered}
\Omega_{d m}=\frac{\Omega_{d m 0}(1+z)^{3(1+\alpha)}}{\left[\Omega_{\Lambda 0}+\Omega_{d m 0}(1+z)^{3(1+\alpha)}\right]^{\alpha /(1+\alpha)} X} \\
\Omega_{\Lambda}=\frac{\Omega_{\Lambda 0}}{\left[\Omega_{\Lambda 0}+\Omega_{d m 0}(1+z)^{3(1+\alpha)}\right]^{\alpha /(1+\alpha)} X} \\
\Omega_{b}=\frac{\Omega_{b 0}(1+z)^{3}}{X}
\end{gathered}
$$

where

$$
X=\left[\Omega_{\Lambda 0}+\Omega_{m 0}(1+z)^{3(1+\alpha)}\right]^{1 /(1+\alpha)}+\Omega_{b 0}(1+z)^{3} .
$$

Finally, as $\Omega_{d m 0}$ and $\Omega_{\Lambda 0}$ are order one quantities, one can easily see that at the time of nucleosynthesis, $\Omega_{\Lambda}$ is negligibly small, and hence the model is not in conflict with known processes at nucleosynthesis.

It is important to realize that there is an explicit interaction between dark matter and dark energy. This can be understood from the energy conservation equation, which in terms of the components can be written as

$$
\dot{\rho}_{d m}+3 H \rho_{d m}=-\dot{\rho}_{\Lambda} .
$$

Thus, the evolution of dark energy and dark matter are coupled so that energy is exchanged between these components (see Refs. $[16,17]$ for earlier work on the interaction between dark matter and dark energy). One can see from Figure 1 , that until $z \simeq 2$, there is essentially no exchange of energy and the $\Lambda$ term is vanishingly small. However, around $z \simeq 2$, the interaction starts to increase, resulting in a substantial growth of the dark energy term at the expense of the dark matter energy. Thus, by around $z \simeq 0.2$, dark energy starts dominating the energy content of Universe. Of course, these redshift values are $\alpha$ dependent and, in Figure 1, $\alpha=0.2$ has been chosen. Nevertheless, the main conclusion is that in this unified model, the interaction between dark matter and dark energy is vanishing small for almost the entire history of the Universe making it indistinguishable from 
the CDM model. As can be clearly seen, the energy transfer has started in the recent past resulting in a significant energy transfer from dark matter to the $\Lambda$-like dark energy. In the next section we show that this energy transfer epoch is the one when dark matter perturbations start departing from its linear behaviour. But before that notice also that Eq. (21) expresses the energy conservation for the background fluid, which is reminiscent of earlier work on varying $\Lambda$ cosmology $y^{5,18,19}$ where the cosmological term decays into matter particles. In here, we have the opposite, as $\alpha$ is always positive, hence the energy transfer is from dark matter to dark energy. This is responsible for the late time dominance of the latter and ultimately to the observed accelerated expansion of the Universe.

\section{Structure Formation}

Aiming to study structure formation, it is interesting to write the 0-0 component of Einstein's equation as

$$
3 H^{2}=8 \pi G\left(\rho_{d m}+\rho_{b}\right)+\Lambda,
$$

where $\Lambda$ is given by

$$
\Lambda=8 \pi G \rho_{\Lambda}
$$

We address now the issue of energy density perturbations. We first write the Newtonian equations for a pressure-less fluid with background density $\rho_{d m}$ and density contrast $\delta_{d m}$, with a source term due to the energy transfer from dark matter to dark energy. Assuming that both, the density contrast $\delta_{d m}$ and the peculiar velocity $v$ are small, that is $\delta_{d m}<<1$ and $v<<u$, where $u$ is the velocity of a fluid element, one can write the Euler, the continuity and the Poisson's equations in the co-moving frame ${ }^{19}$ :

$$
\begin{array}{r}
\ddot{a} x+\frac{\partial v}{\partial t}+\frac{\dot{a}}{a} v=-\frac{\nabla \Phi}{a}, \\
\nabla \cdot v=-a\left[\frac{\partial \delta_{d m}}{\partial t}+\frac{\Psi \delta_{d m}}{\rho_{d m}}\right], \\
\frac{1}{a^{2}} \nabla^{2} \Phi=4 \pi G \rho_{d m}\left(1+\delta_{d m}\right)-\Lambda,
\end{array}
$$

where $\Phi$ is the gravitational potential, and $\Psi$ is the source term in the continuity equation due to the energy transfer between dark matter and 


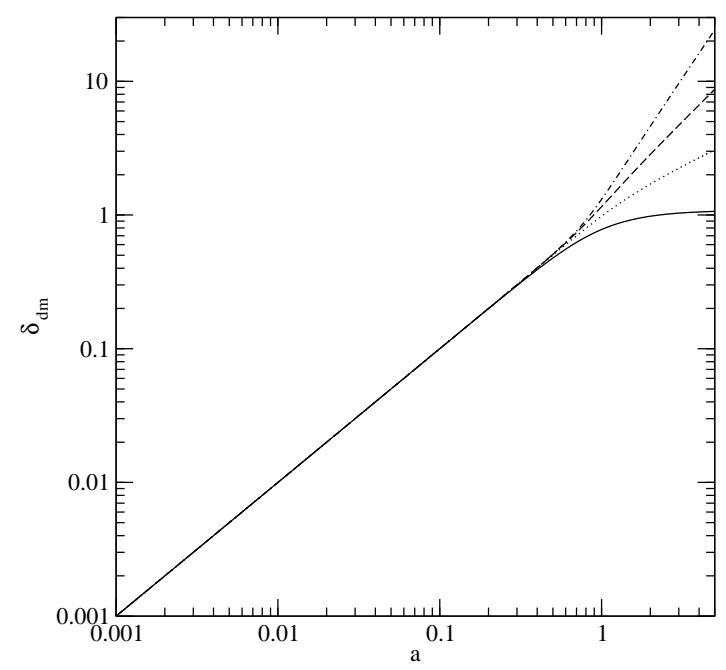

Figure 2. Density profile $\delta_{d m}$ as function of scale factor. The solid, dotted, dashed and dash-dot lines correspond to $\alpha=0,0.2,0.4,0.6$, respectively. It is assumed that $\Omega_{b 0}=0.05, \Omega_{d m 0}=0.25$ and $\Omega_{\Lambda 0}=0.7$.

the cosmological constant-type dark energy. The co-moving coordinate $x$ is related to the proper coordinate $r$ by $r=a x$. In here,

$$
\Psi=-\frac{1}{8 \pi G} \dot{\Lambda}
$$

One expects a perturbation also in the $\Lambda$ term. However, it can be seen from the Euler equation, for a fluid with an equation state of the form $p=w \rho$,

$$
(w+1) \rho\left(\frac{\partial v}{\partial t}+v \cdot \nabla v\right)+w \nabla \rho+(w+1) \rho \nabla \Phi=0
$$

so that, for $w=-1$, it follows that $\nabla \rho=0$, from which implies that this cosmological constant like component is always homogeneous. We should mention that the Euler Eqs. (26) and (28) can have an extra term in the r.h.s. if the velocity of the created $\Lambda$-like particle has a different velocity from the decaying dark matter particle ${ }^{19}$. In this case, the $\Lambda$-like dark energy can have spatial variations which can be neglected for the Newtonian treatment. However, in our case, we are considering only the situation where both the decaying and created particles have the same velocity. 


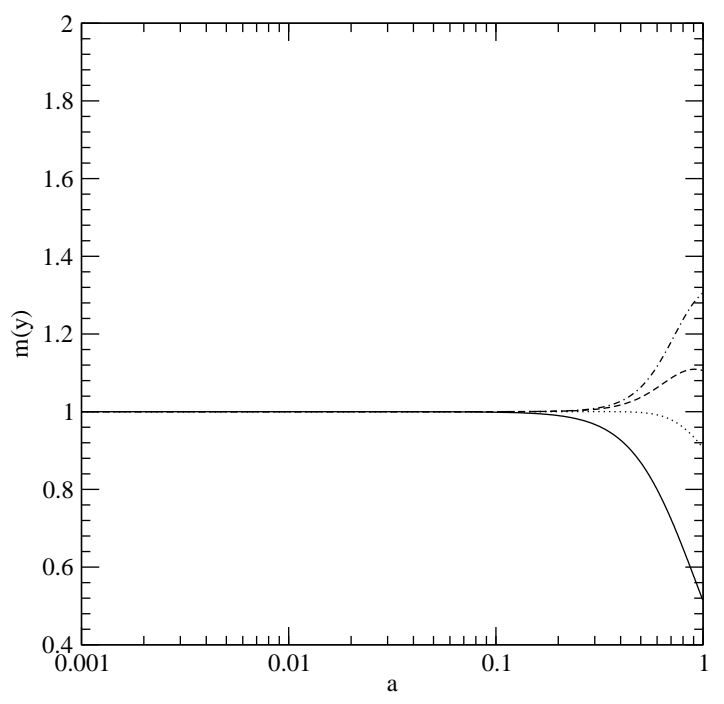

Figure 3. The growth factor $m(y)$ as a function of scale factor a. The solid, dotted, dashed and dash-dot lines correspond to $\alpha=0,0.2,0.4,0.6$, respectively. It is assumed that $\Omega_{b 0}=0.05, \Omega_{d m 0}=0.25$ and $\Omega_{\Lambda 0}=0.7$.

From the divergence of Eq. (24) and using Eqs. (25) and (26), one obtains the small scale linear perturbation equation for the dark matter in the Newtonian limit:

$\frac{\partial^{2} \delta_{d m}}{\partial t^{2}}+\left[2 \frac{\dot{a}}{a}+\frac{\Psi}{\rho_{d m}}\right] \frac{\partial \delta_{d m}}{\partial t}-\left[4 \pi G \rho_{d m}-2 \frac{\dot{a}}{a} \frac{\Psi}{\rho_{d m}}-\frac{\partial}{\partial t}\left[\frac{\Psi}{\rho_{d m}}\right]\right] \delta_{d m}=0$.

One sees that, if $\Psi=0$, that is in the absence of energy transfer, one recovers the standard equation for the dark matter perturbation in the $\Lambda \mathrm{CDM}$ case. One can verify that this occurs for $\alpha=0$. It can also be seen from the above equation that there is no scale dependent term to drive oscillations or to cause any blow up in the power spectrum.

We turn now to the evolution for the baryon perturbations in the Newtonian limit when the scales are inside the horizon. Given that our purpose is to consider the period after decoupling, the baryons are no longer coupled to photons and one can effectively consider baryons as a pressure-less fluid like the dark matter as there is no significant pressure due to Thompson scattering. We assume that there is no interaction between dark energy 
and baryons, which means that the Equivalence Principle is violated as, on its turn, dark matter and dark matter are strongly coupled. Given that it is parameter $\alpha$ that controls this interaction ( $\alpha=0$ means there is no interaction), it is a measure of the violation of the Equivalence Principle. One can also see from the behaviour of $\Psi$, that this violation also starts rather late in the history of the Universe. In the Newtonian limit, the evolution of the baryon perturbation after decoupling for scales well inside the horizon is similar to the one for dark matter however, as described earlier, the source term is absent as there is no energy transfer to or from baryons. Thus, the equation for the evolution of baryon perturbations is given by

$$
\frac{\partial^{2} \delta_{b}}{\partial t^{2}}+2 \frac{\dot{a}}{a} \frac{\partial \delta_{b}}{\partial t}-4 \pi G \rho_{d m} \delta_{d m}=0,
$$

where in the third term in the l.h.s., the contribution from baryons has been dropped as it is negligible compared to the one of dark matter.

It is convenient to define for each component the linear growth function $D(y)$

$$
\delta=D(y) \delta_{0},
$$

where $y=\log (a)$ and $\delta_{0}$ is the initial density contrast (assuming a Gaussian distribution). It is also interesting to consider the so-called growth exponent $m(y)=D^{\prime}(y) / D(y)$, where the prime denotes derivative with respect to the scale factor.

Asymptotically, given that dark matter drives the evolution of the baryon perturbations, then they grow with the same exponent $m(y)$. However, their amplitudes may differ and their ratio corresponds to the so-called bias parameter, $b \equiv \delta_{b} / \delta_{d m}$.

It is of course, phenomenologically interesting to study the behaviour of $\delta_{d m}, m(y)$ and $b$ as function of the scale factor $a$. While solving the differential equations for the linear perturbation, the initial conditions are chosen so that at $a=10^{-3}$, the standard linear solution $D \simeq a$ is reached. In Figure 2, it is shown the linear density perturbation for dark matter, $\delta_{d m}$, as a function of $\alpha$. One sees that, whereas for $\alpha=0$ (the $\Lambda$ CDM case), the perturbation stops growing at late times, for models with $\alpha>0$ the perturbation starts departing from the linear behaviour around $z \simeq 0.25$, the very epoch when the $\Lambda$ term starts dominating (cf. Figure 1). In view of this behaviour, it is tempting to conjecture that, in our unified model, the interaction between dark matter and $\Lambda$-like dark energy is related with 


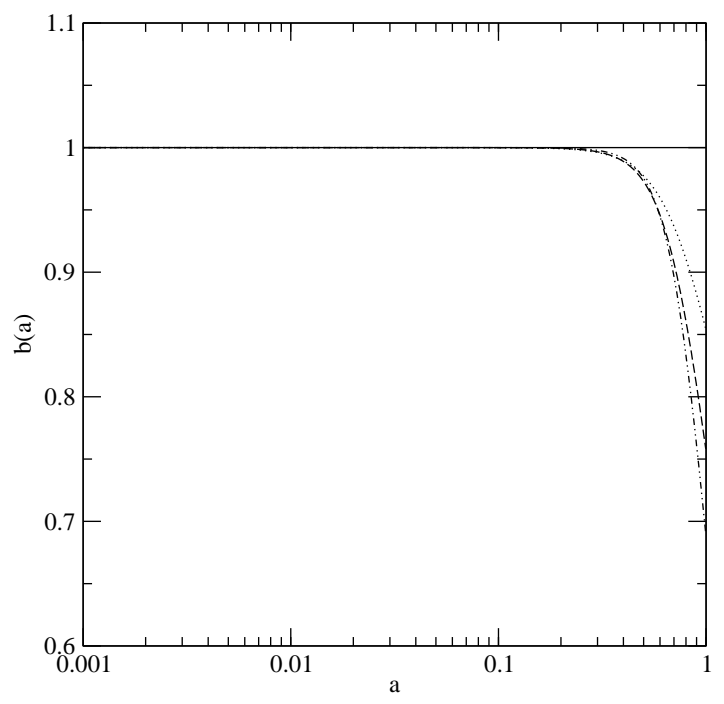

Figure 4. Bias parameter $b$ as a function of the scale factor, $a$. The solid, dotted, dashed and dash-dot lines correspond to $\alpha=0,0.2,0.4,0.6$, respectively. It is assumed that $\Omega_{b 0}=0.05, \Omega_{d m 0}=0.25$ and $\Omega_{\Lambda 0}=0.7$.

structure formation, so that for a sufficiently high density contrast $\left(\delta_{d m}>>\right.$ $1)$, a significant energy transfer from dark matter to dark energy takes place. In any case, our proposal for GCG indicates that there is a connection between structure formation scenario and the dominance of dark energy, a link that ultimately results in the acceleration of the Universe expansion. This feature hints a possible way to understand why $\Omega_{d m} \simeq \Omega_{\Lambda}$ just at recent past, the so-called Cosmic Coincidence problem.

The behaviour of $m(y)$ is also interesting. One can infer from Figure 3 that from $z \simeq 5$ to the present, the growth factor is quite sensitive to the value of $\alpha$. For $\alpha=0.2, m(y)$ increases up to $40 \%$ at present in relation to the $\Lambda \mathrm{CDM}$ case.

Notice that $m(y)$ governs the growth of the velocity fluctuations in the linear perturbation theory as the velocity divergence evolves as $-H a m \delta_{d m}$; it follows then that large deviations of the growth factor with changing $\alpha$ are detectable via precision measurements of large scale structure and associated measurements of the redshift-space power spectrum anisotropy.

In what concerns the bias parameter, its behaviour is shown in Figure 4. From there one can see that it also changes sharply in the recent past as 


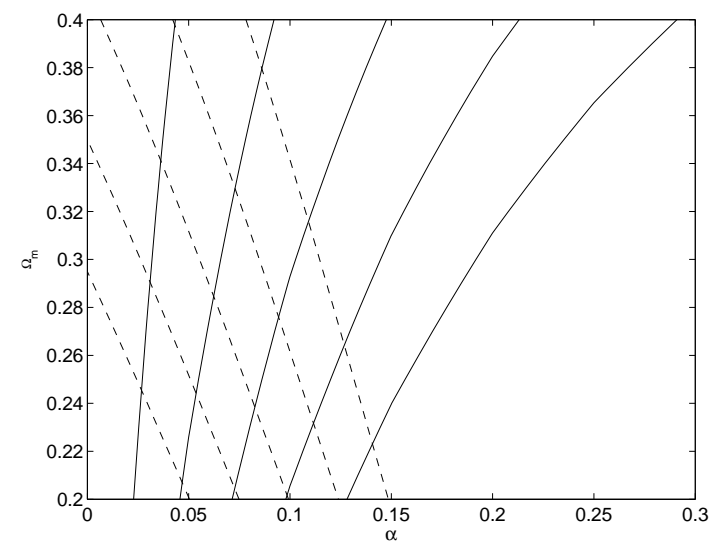

Figure 5. Contours for parameters $b$ and $m$ in the $\Omega_{m}-\alpha$ plane. Solid lines refer to $b$ whereas dashed lines refer to $m$. For $b$, contour values are $0.98,0.96, \ldots, 0.9$ from left to right. For $m$, contour values are $0.6,0.65, \ldots, 0.8$ from left to right.

$\alpha$ increases. This bias extends to all scales consistent with the Newtonian limit, hence being distinguishable from the hydrodynamical or nonlinear bias which takes place only for collapsed objects. Therefore, from the observation of large scale clustering one can distinguish the non-vanishing $\alpha$ case from the $\alpha=0(\Lambda \mathrm{CDM})$ case.

The growth factor and the bias parameter at $z \sim 0.15$ have been recently determined using the $2 \mathrm{DF}$ survey ${ }^{20,21}$. It is found for the redshift space distortion parameter, $\beta=0.49 \pm 0.09$, and for the linear bias, $b=1.04 \pm$ 0.14. Notice that, as $\beta=m / b$, one can obtain $m=0.51 \pm 0.11$. In Figure 5, it is shown contours for $b$ and $m$ in the $\Omega_{m}-\alpha$ plane. From the mentioned observational constraints on $b$ and $m$, one can constrain $\alpha$ to a small but non-zero value $(\alpha \sim 0.1)$. However, it is important to point out that our study refers to the properties of the baryons whereas the observations concern the fraction of baryons that collapsed to form bright galaxies; the relation between the two is still poorly known. As far as parameter $\beta$ is concerned, one should bear in mind that this constraint is obtained in the context of the standard $\Lambda \mathrm{CDM}$ model in order to convert redshift to distance. Thus, a full analysis in the context of the GCG model is still to be performed.

Furthermore, as can be seen from Figure 2, there is no suppression of $\delta_{d m}$ at late times for any positive value of $\alpha$, and hence one should not 
expect the corresponding suppression in the power spectrum normalization, $\sigma_{8}$, for the total matter distribution. This was one major problem in the previous GCG model approach which, as pointed out in Ref. [14], cannot be solved even after the inclusion of baryons. In the approach developed in Ref. [15] and described here, one can overcome this difficulty.

Another interesting cosmological test for our model is the study of the $M / L$ ratio for clusters of galaxies. The most recent average value ${ }^{22}, \Omega_{m}=$ $0.17 \pm 0.05$, has been extracted from the observation of 21 clusters with $z \sim 1$. The fact that nearby cluster data seem to prefer smaller values for $\Omega_{m}$ than the one obtained from WMAP data, can be interpreted as a signal in favour of a decaying dark matter model such as the GCG.

\section{Conclusions}

In this contribution, we have presented a setup where the GCG has been decomposed in two interacting components. The first one behaves as dark matter since it is pressure-less. The second one has an equation of state, $p_{X}=\omega_{X} \rho_{X}$. It has been shown that $\omega_{X} \leq-1$. Thus, once phantom-like behaviour is excluded the decomposition is unique. Apparently the model does not look different from the interacting quintessence models where one has two different interacting fluids; however, an interesting feature of our proposal is that it can be described through a single fluid equation. Hence, as far the background cosmology is concerned, we have an unified GCG fluid behaving as dark matter in the past and as a dark energy in the present. Nevertheless, when studying structure formation in this model one should consider it as an interacting mixture of two fluids to achieve a proper description. In any unified model, one expects an entangled mixture of interacting dark matter and dark energy. In the case of the GCG, we can uniquely identify the components of this mixture and the interaction. Moreover, we find that one does not need anything besides an evolving cosmological term to describe dark energy. This is consistent with recent studies that show that a combination of WMAP data and observations of high redshift supernovae can be described via a cosmological constant-like dark energy ${ }^{23}$. One can also consider the GCG as a decaying dark matter model where the decay product is a cosmological constant.

Obviously it remains to be seen how one can obtain such a decaying dark matter model from a fundamental theory. Given the fact that the GCG equation of state arises from a generalized Born-Infeld action, it is possible that D-brane physics can shed some light into this issue (see eg. 
Ref. [24]).

Furthermore, we have demonstrated that in the context of our setup, the so-called dark energy dominance is related with the time when matter fluctuations become large $\left(\delta_{d m}>1\right)$, a possibility has actually been

previously conjectured ${ }^{25}$. Moreover, we have shown that in what concerns structure formation, the linear regime $\left(\delta_{d m} \sim a\right)$ is valid till fairly close to the present, meaning that at the time structure formation begins, $z_{c} \simeq 10$, the influence of the dark energy component was negligible and that clustering occurs very much like in the CDM model. We have shown that the growth factor as well as the bias parameter have a noticeable dependence on the $\alpha$ parameter. We have implemented a model which exhibits a violation of the Equivalence Principle, as dark energy and baryons are not directly coupled. This may turn out to be an important observational signature of our approach.

\section{Acknowledgments}

It is a pleasure to thank Maria Bento, Anjan Sen, Somasri Sen and Pedro Silva for sharing the fun on the research of the GCG properties.

\section{References}

1. A. Kamenshchik, U. Moschella and V. Pasquier, Phys. Lett. 511 (2001) 265.

2. M.C. Bento, O. Bertolami and A.A. Sen, Phys. Rev. D66 (2002) 043507.

3. See e.g. M.C. Bento and O. Bertolami, Gen. Relat. and Gravitation 31 (1999) 1461; M.C. Bento, O. Bertolami and P.T. Silva, Phys. Lett. B498 (2001) 62.

4. B. Ratra and P.J.E. Peebles, Phys. Rev. D37 (1988) 3406; Ap. J. Lett. 325 (1988) 117; C. Wetterich, Nucl. Phys. B302 (1988) 668; R.R. Caldwell, R. Dave and P.J. Steinhardt, Phys. Rev. Lett. 80 (1998) 1582; P.G. Ferreira and M. Joyce, Phys. Rev. D58 (1998) 023503; I. Zlatev, L. Wang and P.J. Steinhardt, Phys. Rev. Lett. 82 (1999) 986; P. Binétruy, Phys. Rev. D60 (1999) 063502; J.E. Kim, JHEP 9905 (1999) 022; J.P. Uzan, Phys. Rev. D59 (1999) 123510; T. Chiba, Phys. Rev. D60 (1999) 083508; L. Amendola, Phys. Rev. D60 (1999) 043501; O. Bertolami and P.J. Martins, Phys. Rev. D61 (2000) 064007; Class. Quantum Gravity 18 (2001) 593; A.A. Sen, S. Sen and S. Sethi, Phys. Rev. D63 (2001) 107501; A.A. Sen and S. Sen, Mod. Phys. Lett. A16 (2001) 1303; A. Albrecht and C. Skordis, Phys. Rev. Lett. 84 (2000) 2076; Y. Fujii, Phys. Rev. D61 (2000) 023504; M.C. Bento, O. Bertolami and N.C. Santos, Phys. Rev. D65 (2002) 067301.

5. M. Bronstein, Phys. Zeit. Sowejt Union 3 (1933) 73; O. Bertolami, Il Nuovo Cimento 93B (1986) 36; Fortschr. Physik 34 (1986) 829; M.Ozer and M.O Taha, Nucl. Phys. B287 (1987) 776.

6. M.C. Bento, O. Bertolami and A.A. Sen, Phys. Lett. B575 (2003) 172; Phys. 
Rev. D67 (2003) 063003; Gen. Relat. and Gravitation 35 (2003) 2063; D. Caturan and F. Finelli, Phys. Rev. D68 (2003) 103501; Amendola, F. Finelli, C. Burigana and D. Caturan, JCAP 0307 (2003) 005.

7. J.C. Fabris, S.B.V. Gonçalves and P.E. de Souza, astro-ph/0207430; A. Dev, J.S. Alcaniz and D. Jain, Phys. Rev. D67 (2003) 023515; V. Gorini, A. Kamenshchik and U. Moschella, Phys. Rev. D67 (2003) 063509; M. Makler, S.Q. de Oliveira and I. Waga, Phys. Lett. B555 (2003) 1; J.S. Alcaniz, D. Jain and A. Dev, Phys. Rev. D67 (2003) 043514.

8. P.T. Silva and O. Bertolami, Ap. J. 599 (2003) 829; A. Dev, D. Jain and J.S. Alcaniz, Astron. Astrophys. 417 (2004) 847.

9. J.L. Tonry et al. Ap. J. 594 (2003) 1; B.J. Barris et al., Ap. J. 602 (2004) 571; A.G. Riess et al. [Supernova Search Team Collaboration], Ap. J. 607 (2004) 665.

10. O. Bertolami, A.A. Sen, S. Sen and P.T. Silva, Mont. Not. R. Astr. Soc. 353 (2004) 329 .

11. M.C. Bento, O. Bertolami, A.A. Sen, and N.C. Santos, Phys. Rev. D71 (2005) 063501.

12. N. Bilić, G.B. Tupper and R.D. Viollier, Phys. Lett. B535 (2002) 17; J.C. Fabris, S.B.V. Gonçalves and P.E. de Souza, Gen. Relat. and Gravitation 34 (2002) 53.

13. O. Bertolami, astro-ph/0403310.

14. H. Sandvik, M. Tegmark, M. Zaldarriaga and I. Waga, Phys. Rev. D69 (2004) 123524 .

15. M.C. Bento, O. Bertolami and A.A. Sen, Phys. Rev. D70 (2004) 083519.

16. L. Amendola, Phys. Rev. D62 (2000) 043511; D. Tocchini-Valentini and L Amendola, Phys. Rev. D65 (2002) 063508.

17. L.P. Chimento, A.S. Jakubi, D. Pavón and W. Zimdahl, Phys. Rev. D67 (2003) 083513.

18. K. Freese, F.C. Adams, J.A. Frieman and E. Mottola, Nucl. Phys. B287 (1987) 797.

19. R.C. Arcuri and I. Waga, Phys. Rev. D50 (1994) 2928.

20. E. Hawkins et.al., Mont. Not. R. Astr. Soc. 346 (2003) 78.

21. L. Verde et.al., Mont. Not. R. Astr. Soc. 335 (2002) 432.

22. N. Bahcall and J.M. Comerford, Ap. J. Lett. 565 (2002) L5.

23. H.K. Jassal, J.S. Bagla and T. Padmanabhan, Mont. Not. R. Astr. Soc. Lett. 356 (2005) L11.

24. R. Neves and C. Vaz, Phys. Lett. B568 (2003) 153.

25. U. Alam, V. Sahni, T.D. Saini and A.A. Starobinsky, Mont. Not. R. Astr. Soc. 354 (2004) 275. 


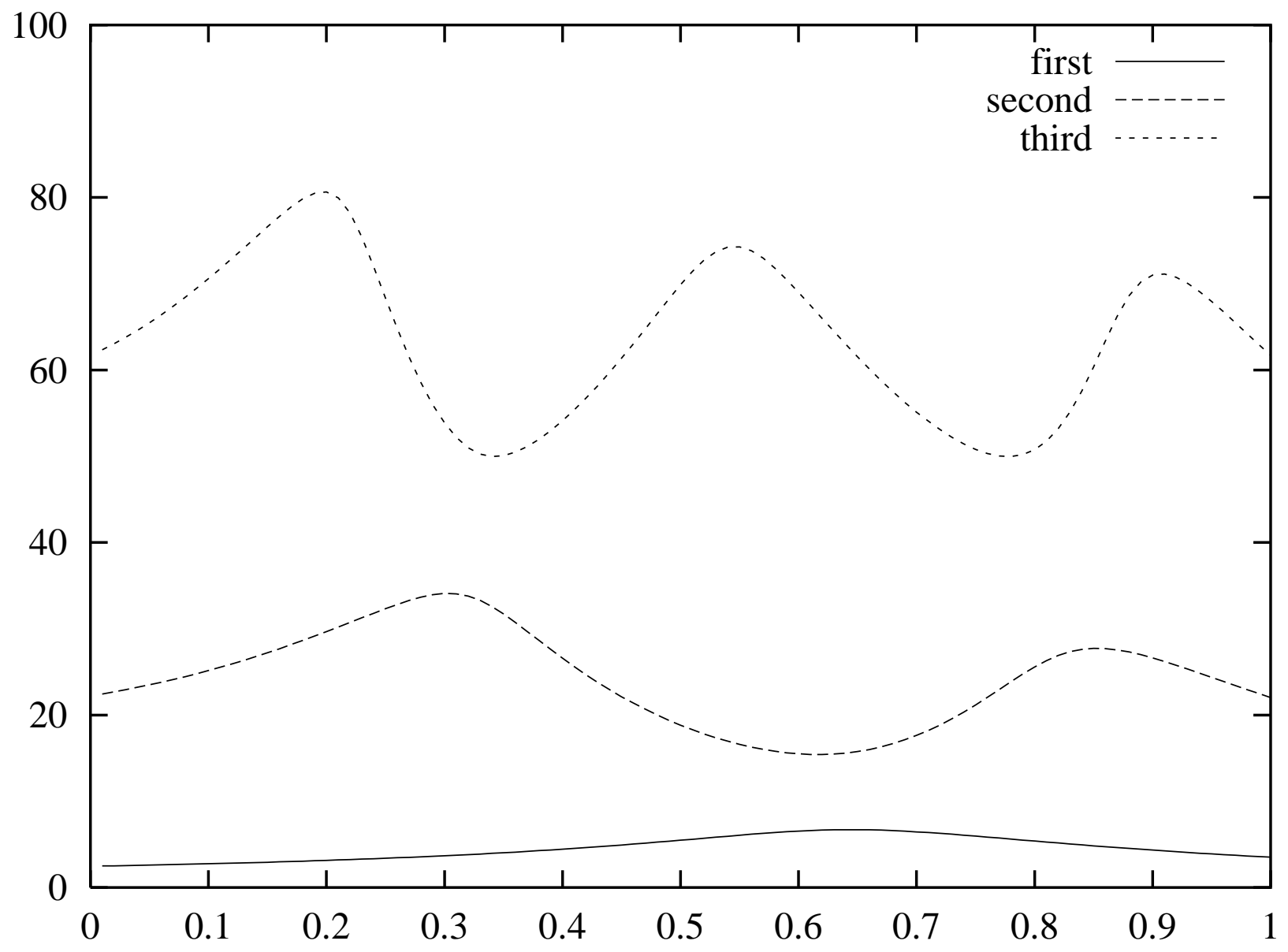

